\title{
Epithelioid Angiosarcoma in Femur: A Case Presentation
}

\author{
Yunus Oc ${ }^{\mathrm{a}}$, Bekir Eray Kilinc ${ }^{\mathrm{b}, \mathrm{c}}$, Rodi Ertugrul ${ }^{\mathrm{a}}$, Hasan Basri Sezer ${ }^{\mathrm{a}}$, \\ Osman Tugrul Eren ${ }^{\mathrm{a}}$
}

\begin{abstract}
Primary malignant vascular tumors which constitute less than $1 \%$ of the primary bone tumors are very rarely encountered. Epithelioid angiosarcoma is a very uncommon subtype of angiosarcomas presenting with an epithelioid phenotype and mimicking carcinomas clinically. These tumors select mostly extra-skeletal locations but rarely bones as their medium. A 31-year-old male patient applied to our clinic with a nonspecific left hip pain. X-ray evaluation revealed a cystic expansile mass in the trochanteric region of the femur extending to the femoral neck. Computed tomography examination proved that the mass was cystic and $7 \mathrm{~cm}$ in its greatest width and contained septum. Moreover, magnetic resonance imaging examination was reported as a mass resembling aneurysmal bone cyst. The final diagnosis of epithelioid angiosarcoma was made by histological examination and biopsy of the mass. The excised specimen was examined further to verify the preoperative diagnosis.
\end{abstract}

Keywords: Angiosarcoma; Bone; Vascular tumor

\section{Introduction}

Angiosarcoma is a type of malignant mesenchymal tumor which was characterized with the presence of endothelial differentiation of the tumoral cells. The bone is in an unusual localization for angiosarcoma, and skeletal lesions are clinically aggressive. High local recurrence rate, lymph node and systemic metastasis were reported [1]. It presents mostly in the second and seventh decades of life [1]. The pathological diagnosis may require several biopsies and radiological support for the certain diagnosis.

\section{Case Report}

A 31-year-old man applied to our outpatient clinic with left hip

Manuscript submitted October 23, 2017, accepted November 24, 2017

aOrthopaedics and Traumatology Department, Sisli Hamidiye Etfal Training and Research Hospital, Istanbul, Turkey

bOrthopaedics and Traumatology Department, Golhisar State Hospital, Burdur, Turkey

${ }^{\mathrm{c}}$ Corresponding Author: Bekir Eray Kilinc, Fatih Mahallesi Golhisar Devlet Hastanesi, Burdur 15400, Turkey. Email: dreraykilinc@gmail.com

doi: https://doi.org/10.14740/wjon1066e pain that lasted for 6 months. In the X-ray examination, an expansile, multilobulated and cystic mass located in the proximal part of left femur was observed. The computed tomography (CT) examination revealed a multilobulated, lytic mass of 7 $\mathrm{cm}$ in diameter containing thin hyperdense septum extending to the greater trochanter and neck region, which was reported as a complicated cystic mass (Fig. 1).

Magnetic resonance imaging (MRI) examination was carried out to further examine this intramedullary mass. There was an intramedullary heterogeneous cystic mass appearance in the proximal part of the left femur (Fig. 2). The posterior cortex was destroyed and there was soft tissue extension resembling aneurysmal bone cyst, and Jamshidi needle biopsy was advised.

The first biopsy was not definitive in describing the sample as partially degenerated bone fragment containing low degree chronic inflammation and focal fibrochondroid tissue. The second biopsy was carried out to rule out malignancy. The second biopsy revealed osteoclastic giant cells between bone lamella and proliferated vascular tissue with epithelioid morphology containing atypical fusiform cell proliferation. The immunohistochemical analysis with CD31 stain was positive supporting the diagnosis of angiosarcoma (Fig. 3a-c).

The patient was treated with wide resection of the left proximal femur and partial replacement of the left hip with tumor resection prosthesis (Fig. 4a). The excised proximal femur was examined pathologically to validate the diagnosis and to check the surgical borders (Fig. 4b). The surgical borders were reported to be clean. The patient was undergone a regimen of chemotherapy and radiotherapy postoperatively. X-rays and positron emission tomography (PET) scan in the 18th month postoperatively revealed no metastasis. There was no complication or surgical site infection. The patient returned to the daily life, and has been followed up regularly in every 3 months.

\section{Discussion}

Primary vascular bone tumors are extremely rare. Angiosarcoma is a malignant vascular endothelial tumor which selects soft tissue locations mostly. Primary angiosarcoma of bone constitutes less than $1 \%$ of bone tumors [2]. These tumors exert male predominance. The angiosarcoma of bone may be localized in both short and long bones of the skeleton; femur, tibia and humerus are the most probable locations [3].

The etiology of malignant vascular bone tumors is unclear. The early diagnosis with biopsy may be the only chance of the patient to survive. The definitive diagnosis is done with 


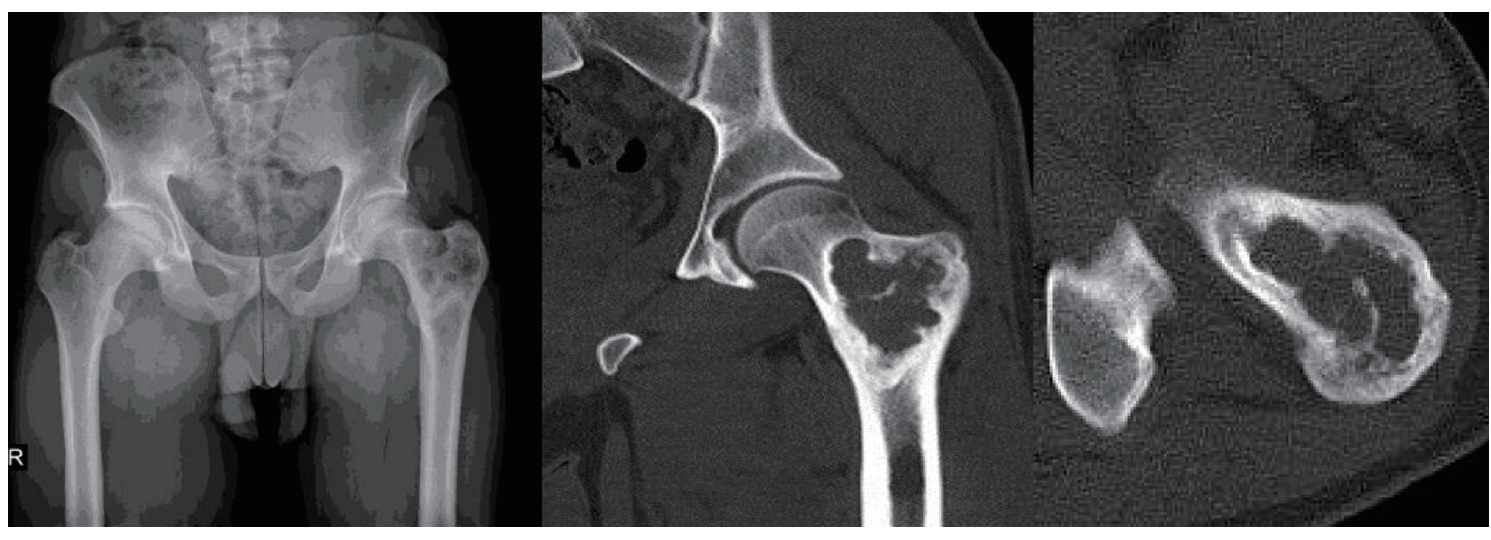

Figure 1. Preoperative X-ray and CT views of the mass. CT: computed tomography.

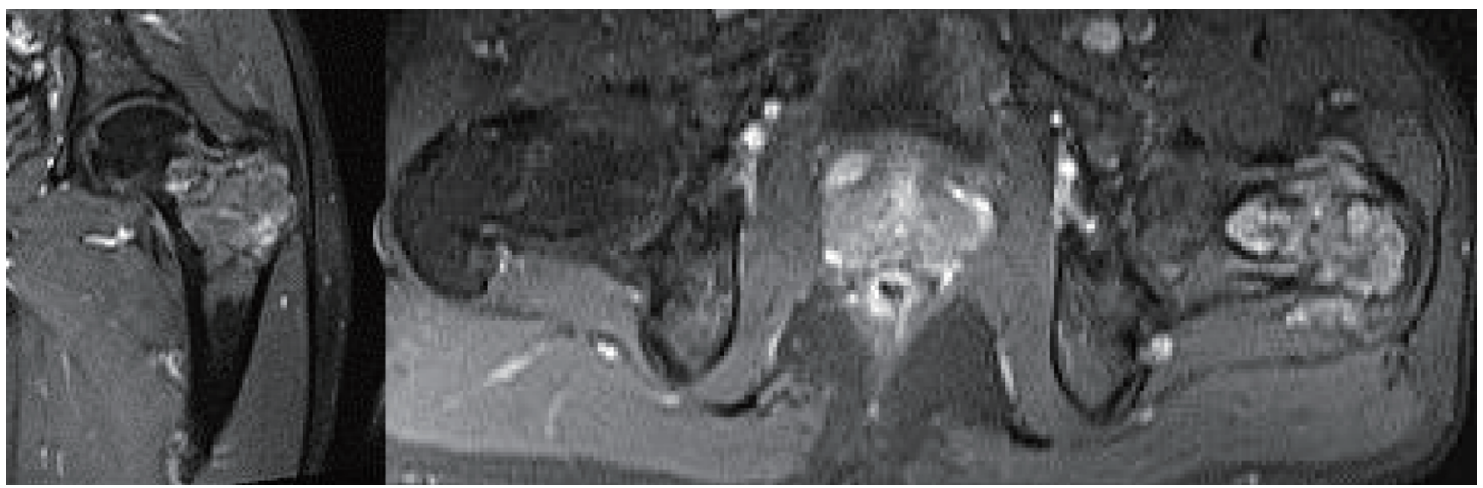

Figure 2. Preoperative MRI view. MRI: magnetic resonance imaging.

histological and immunohistochemical tests [4]. The key of the immunohistochemical examination is the application of vascular markers for the diagnosis of epithelioid angiosarcoma. The most sensitive vascular marker is CD31 [5]. Factor VIII and CD34 markers are the other useful markers for diagnostic studies. Desphande et al examined 10 patients and proved $75 \%$ patients as positive with factor VIII and $40 \%$ patients as positive with CD34 markers [5]. Our patient was stained positive with the most sensitive marker, CD31.

Epithelioid angiosarcoma hosts a potential risk of delayed diagnosis due to its very rare occurance and misinterpretations as metastatic carcinoma mostly as renal cell carcinoma. The other possible options of misdiagnoses are vascular tumors mostly the hemangioma and low grade angiosarcoma. Basil- lary angiomatosis is a process characterized with vascular proliferation, epithelioid endothelial cells showing cytological atypia. The differential diagnosis of basillary angiomatosis is done easily in the presence of bacterial clusters and neutrophilic proliferation.

Different from common attitude of soft tissue sarcomas, which selectively metastasize to lungs, epithelioid angiosarcoma metastasize to lymph nodes and solid organs. Lungs, bone, soft tissues and skin are the possible locations of metastasis. More than $50 \%$ of patients diagnosed as epithelioid angiosarcoma are lost in 3 years $[1,5]$. The treatment alternatives are similar to bone sarcomas; amputation, radical resection combined with radiotherapy and/or chemotherapy [6-8].

Fluorodeoxyglucose PET (FDG-PET) is a very useful de-
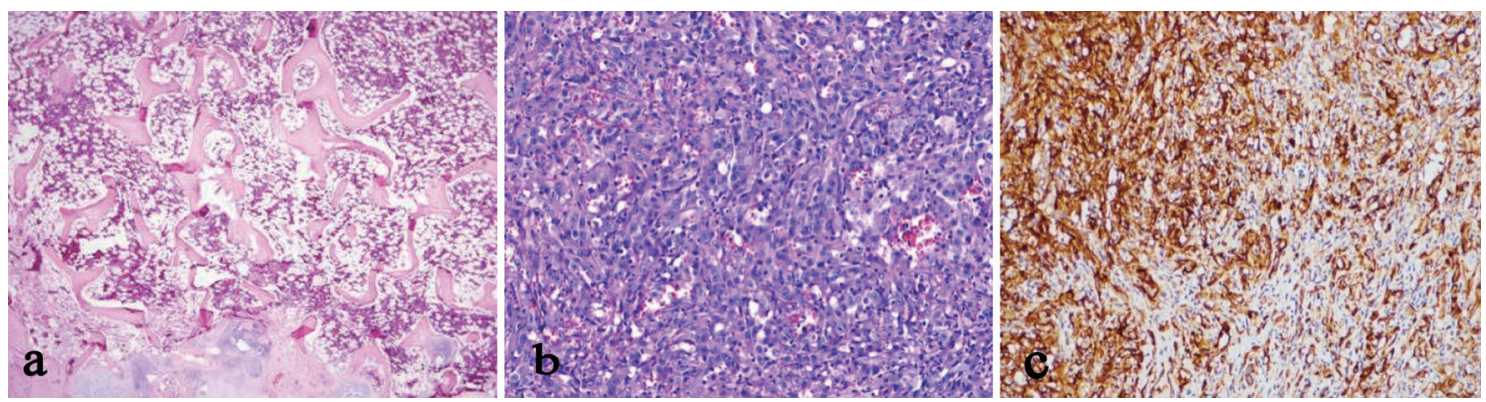

Figure 3. (a) Normal bone histology, $\times 40$ magnification. (b) Hematoxylin-eosin stain. (c) Immunohistochemical stain with CD31. 


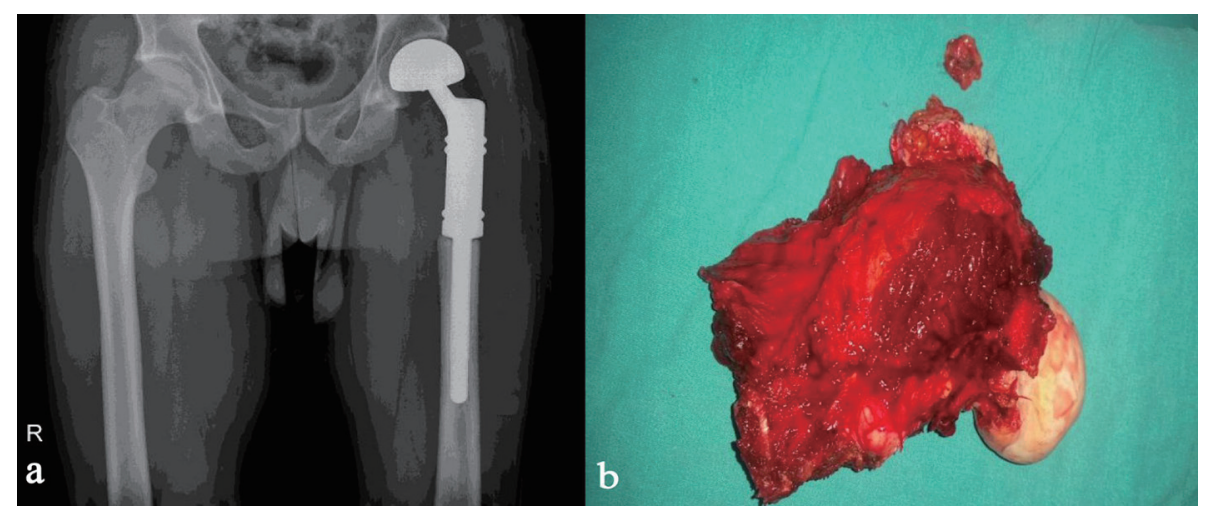

Figure 4. (a) Postoperative X-ray. (b) Excised sample of bone.

vice as a postoperative follow-up tool to rule out lymph node metastasis $[9,10]$. Our patient is screened with FDG-PET in 3 monthly periods and he is in the 18th month postoperatively free of local recurrence or metastasis.

\section{Conclusions}

Bone angiosarcoma is one of the diagnoses that should be kept in mind in cases of difficulty in diagnosis. With early diagnosis and good surgical treatment, life expectancy is increasing.

\section{Conflict of Interest}

The authors declare that there is no conflict of interest regarding the publication of this article.

\section{References}

1. Meis-Kindblom JM, Kindblom LG. Angiosarcoma of soft tissue: a study of 80 cases. Am J Surg Pathol. 1998;22:683-697.

2. Wenger DE, Wold LE. Malignant vascular lesions of bone: radiologic and pathologic features. Skeletal Radiol.
2000;29:619-631.

3. Unni KK, Inwards CY, Bridge JA, Kindblom L-G, Wold LE. Tumors of the Bones and Joints. AFIP atlas of tumor pathology. 2006;10:266-273.

4. Markaki S, Kokka H, Kyparidou E, Bouropoulou V. Primary vascular bone sarcomas. A clinicopathological and immunohistochemical study of two cases. Arch Anat Cytol Pathol. 1990;38:163-167.

5. Deshpande V, Rosenberg AE, O'Connell JX, Nielsen GP. Epithelioid angiosarcoma of the bone: a series of 10 cases. Am J Surg Pathol. 2003;27:709-716.

6. Hasegawa T, Fujii Y, Seki K, Yang P, Hirose T, Matsuzaki $\mathrm{K}$, et al. Epithelioid angiosarcoma of bone. Hum Pathol. 1997;28:985-988.

7. Mittal S, Goswami C, Kanoria N, Bhattacharya A. Postirradia- tion angiosarcoma of bone. J Cancer Res Ther. 2007;3:96-99.

8. Saglik Y, Yildiz Y, Atalar H, Basarir K. Primary angiosarcoma of the fibula: a case report. Acta Orthop Belg. 2007;73:799-803.

9. Sakamoto A, Takahashi Y, Oda Y, Iwamoto Y. Aggressive clinical course of epithelioid angiosarcoma in the femur: a case report. World J Surg Oncol. 2014;12:281.

10. Deyrup AT, Montag AG. Epithelioid and epithelial neoplasms of bone. Arch Pathol Lab Med. 2007;131:205216. 